\title{
Colonização micorrízica, densidade de esporos e diversidade de fungos micorrízicos arbusculares em solo de Cerrado sob plantio direto e convencional
}

\section{Mycorrhizal colonization, spore density and diversity of arbuscular mycorrhizal fungi in Cerrado soil under no-till and conventional tillage systems}

\author{
Guilherme Augusto Robles Angelini ${ }^{1}$; Arcângelo Loss ${ }^{2 *}$; Marcos Gervasio Pereira²; \\ José Luiz Rodrigues Torres ${ }^{3}$; Orivaldo José Saggin Júnior ${ }^{4}$
}

\section{Resumo}

\begin{abstract}
A busca da sustentabilidade na produção agrícola através de manejos conservacionistas, como o sistema plantio direto, tem favorecido os processos bioquímicos do solo como dos fungos micorrízicos arbusculares (FMAs), os quais promovem um aumento expressivo da superfície específica de absorção do sistema radicular das plantas. Sendo assim, o objetivo deste trabalho foi avaliar a colonização micorrízica, densidade de esporos e a diversidade de FMAs na rizosfera de milho e soja cultivados sob plantio direto com diferentes plantas de cobertura e comparadas a uma área de preparo convencional e pousio, em Uberaba, MG. As culturas de milho e soja foram rotacionadas com milheto, crotalária e braquiária. O delineamento experimental foi em blocos casualizados, com parcelas subdivididas. $\mathrm{O}$ experimento foi implantado em 2000 e, em 2007 avaliou-se a colonização radicular por FMAs (COL) e densidade de esporos no solo (ESP) nas profundidades de 0,0-0,05 m e 0,05-0,10 m, já a avaliação da diversidade de FMAs foi na profundidade de $0,0-0,10 \mathrm{~m}$. Verificou-se que houve efeito significativo das culturas sobre COL e ESP. Entretanto, o efeito das coberturas e do manejo foi verificado apenas em 0,0$0,05 \mathrm{~m}$. As raízes de milho apresentam maiores porcentagens de COL e ESP quando comparado com a soja, para as coberturas de braquiária e milheto. A colonização micorrízica da soja e do milho na área de Cerrados foi acima de $80 \%$ para soja e $95 \%$ para o milho. O sistema de preparo convencional do solo apresentou o menor número de espécies de FMAs em relação às coberturas de milheto e braquiária, em sistema plantio direto com milho e soja. A análise de componentes principais com algumas variáveis químicas, físicas e biológicas do solo evidencia a separação das áreas avaliadas, sendo as variáveis COL e ESP eficientes na separação das áreas em cultivo, para as condições do presente estudo.
\end{abstract}

Palavras-chave: Sistema plantio direto, culturas do milho e da soja, plantas de cobertura, indicadores de qualidade do solo

\footnotetext{
${ }^{1}$ Eng $^{\mathrm{o}} \mathrm{Agr}^{\mathrm{o}}$, Doutorando em Fitotecnia, Universidade Federal Rural do Rio de Janeiro, UFRRJ, Dept ${ }^{\mathrm{O}}$ de Fitotecnia, Seropédica, RJ. E-mail: gara_agr@hotmail.com

${ }^{2}$ Eng $^{\circ}$ Agr $^{\circ}$, Prof. Dr. em Ciência do Solo, Instituto de Agronomia, UFRRJ, Dept ${ }^{\circ}$. Solos, Seropédica, RJ. E-mail: arcangeloloss@ yahoo.com.br; gervasio@ufrrj.br

${ }^{3}$ Eng $^{\circ}$ Agr $^{\circ}$, Prof. Dr. do Instituto Federal de Educação, Ciência e Tecnologia do Triângulo Mineiro, Campus Uberaba, Uberaba, MG. E-mail: jlrtorres@cefetuberaba.edu.br

${ }^{4}$ Eng $^{\circ}$ Agr $^{\circ}$, Pesquisador Dr. da Embrapa Agrobiologia, Seropédica, RJ. E-mail: saggin@cnpab.embrapa.br

* Autor para correspondência
} 


\begin{abstract}
The quest for sustainability in agricultural production through conservation management practices such as no-tillage, has favored the biochemical processes of soil, such as soil arbuscular mycorrhizal fungi (AMFs), which promote a significant increase in specific surface absorption of the root system of plants. The objective of this study was to evaluate the root colonization, spore density and diversity of AMFs in rhizosphere of corn and soybean grown under no-tillage with different cover crops and compared an area conventional tillage and fallow, in Uberaba, state of Minas Gerais. The corn and soybeans were rotated with millet, crotalaria and brachiaria. The experimental design was randomized blocks, with split plots. The experiment was established in 2000, and in 2007 assessed the colonization (COL) and spore density (ESP) $(0.0-0.05 \mathrm{~m}$ and $0.05-0.10 \mathrm{~m})$. In the layer of $0.0-0.10 \mathrm{~m}$ evaluated the diversity of AMFs. It was found that there was a strong effect of culture on COL and ESP. However, the effect of the covers and management was seen only in $0.0-0.05 \mathrm{~m}$. The roots of corn has a higher percentages of COL and ESP compared with soybeans, for the coverage Brachiaria and millet. The mycorrhizal colonization of soybean and maize in Cerrado area was up $80 \%$ for soybeans and up $95 \%$ for corn. Conventional tillage soil the lowest number of AMFs species in relation to the coverage of millet and Brachiaria in notill corn and soybeans. The principal components analysis with some chemical, physical and biological factors of soil shows the separation of the areas assessed, and the biological component (COL and ESP) in the efficient separation of the areas under cultivation, for the conditions of this study.
\end{abstract}

Key words: no-tillage system, corn culture, soybens culture, cover crops, indicators of soil quality

\section{Introdução}

A busca da sustentabilidade na produção agrícola utilizando-se de manejos conservacionistas, como o sistema plantio direto que se baseia na rotação de culturas e no uso de plantas de cobertura (gramíneas e leguminosas) para aumento e/ou manutenção da palhada sobre o solo, tem favorecido os processos bioquímicos do solo resultantes da atividade microbiana, tendo efeitos sobre as propriedades físicas e químicas do solo e reflexos sobre o desenvolvimento das plantas, produtividade agrícola e qualidade ambiental (SOUZA et al., 2006; FIGUEIREDO et al., 2007; RESCK et al., 2008; SIQUEIRA NETO et al., 2009; PEREIRA et al., 2010; LOSS et al., 2011; ROSA et al., 2011).

Estudos de manejo do solo no Cerrado vêm sendo conduzidos com o objetivo de desenvolver estratégias para reduzir o impacto das atividades agrícolas sobre esse ambiente. As altas temperaturas e o manejo inadequado do solo, como o plantio convencional e uso em monocultivo, podem levar a uma diminuição dos estoques de carbono de origem orgânica (FONTANA et al., 2006; MORETI et al., 2007; TORRES; PEREIRA; FABIAN, 2008; SIQUEIRA NETO et al., 2009; PEREIRA et al., 2011), pois afetam as taxas metabólicas dos microrganismos nos processos de decomposição dos resíduos vegetais e da matéria orgânica do solo (MOS) (LA SCALA JUNIOR; BOLONHEZI; PEREIRA, 2006).

O sistema plantio direto, também denominado de sistema de semeadura direta na palha, é uma prática cultural que visa à recuperação e/ou manutenção dos atributos físicos, químicos e biológicos dos solos (MORETI et al., 2007; DENARDIN et al., 2008; SILVA et al., 2009; LOSS, 2011; SCHNEIDER et al., 2011) e, hoje representa o manejo conservacionista mais utilizado no Brasil (DENARDIN et al., 2008; LOSS et al., 2011; PEREIRA et al., 2011).

A manutenção dos resíduos culturais na superfície do solo, o não-revolvimento do solo e a rotação de culturas no sistema de plantio direto proporcionam uma decomposição lenta e gradual do material vegetal depositado que, associado com a fração mineral do solo, favorece o acúmulo da MOS (DIEKOW et al, 2005, 2006; PEREIRA et al., 2011; LOSS et al., 2011). A permanência da cobertura morta sobre a superfície do solo minimiza os riscos de erosão (CARVALHO et al., 2003; BABALOLA; OSHUNSANYA; ARE, 2007; DENARDIN et 
al., 2008), preserva a umidade do solo, mantendo um equilíbrio físico-químico de nutrientes para as culturas (ZHANG et al., 2005; LI et al., 2008). Ao contrário do que ocorre no sistema de preparo convencional, o solo em sistema plantio direto acumula mais carbono e nitrogênio (BAYER, et al., 2003; WOHLENBERG et al., 2004; PEREIRA et al., 2010; 2011) e, o não-revolvimento do solo favorece a biodiversidade, melhorando a porosidade e a proliferação de inimigos naturais de pragas e doenças (FERNANDES, 1995; ANDERSON, 2008; LOSS et al., 2009b).

O sistema de plantio direto é um importante manejo para a manutenção e recuperação da capacidade produtiva de solos (TORRES et al., 2005, TORRES; PEREIRA; FABIAN, 2008; PEREIRA et al., 2010) e que pode também influenciar os fungos micorrízicos arbusculares (FMAs), já que estão presentes em solos e são diretamente influenciados pelo manejo e fertilidade destes (LOSS et al., 2009a). Estes fungos têm papel essencial nos ecossistemas, beneficiando as plantas pelo aumento da absorção de água e nutrientes e protegendo-as contra estresses químicos, físicos e biológicos (AUGÉ, 2001; BERBARA; SOUZA; FONSECA, 2006).

$\mathrm{O}$ interesse em estudos sobre a biologia, diversidade e atividade dos microorganismos do solo está cada vez maior, principalmente com os que cumprem função na ciclagem de nutrientes e/ou produtividade dos ecossistemas, como por exemplo, os FMAs (BERBARA; SOUZA; FONSECA, 2006). Levando em consideração a importância ecológica destes fungos, reconhecidos como um componente integral e fundamental na construção e estabilidade de ecossistemas de todo o planeta (VAN DER HEIJDEN; KUYPER, 2003), a estrutura da sua comunidade é um importante indicador de qualidade do solo (JEFFRIES; BAREA, 2001; CORKIDI et al., 2002).

Sabe-se que a diversidade da comunidade de FMAs está diretamente relacionada à diversidade da cobertura vegetal (VAN DER HEIJDEN et al., 1998;
LOSS et al., 2009a; MIRANDA; SILVA; SAGIN JUNIOR, 2010). Os FMAs causam impactos que vão desde suas relações com plantas (processos de absorção de nutrientes), com comunidades vegetais (influenciando sua diversidade e abundância) e, finalmente, com processos relacionados à estabilidade de ecossistemas, ao participarem de forma ativa e significante na dinâmica do carbono e agregação do solo (BERBARA; SOUZA; FONSECA, 2006). Sendo assim, tornase importante manejar a diversidade da cobertura vegetal para obter populações diversificadas de FMAs, conseguindo-se o máximo potencial das espécies micorrízicas em prol das espécies vegetais (ANTONIOLLI et al., 2002).

Estes microrganismos ocupam um importante nicho ecológico na agricultura sustentável, pois são influenciados pelas práticas de manejo do solo como adubações, tendo, porém a incidência de algumas espécies de FMAs reduzidas pela monocultura extensiva e uso indiscriminado de agrotóxicos (SIQUEIRA; FRANCO, 1988). Isso adquire relevância no bioma Cerrado, que geralmente apresentam solos com níveis bastante críticos de macronutrientes, com elevada concentração de alumínio e também elevado nível de acidez, acarretando em baixos valores de $\mathrm{pH}$ (CORDEIRO et al., 2005; RESCK et al., 2008; LOSS, 2011).

Desta forma, o presente estudo teve o objetivo de avaliar a colonização micorrízica, a densidade de esporos e a diversidade de FMAs na rizosfera de plantas de milho e soja cultivadas em sistema plantio direto sob cobertura de milheto, braquiária e crotalária, e comparado a área de preparo convencional e pousio, em Uberaba, MG.

\section{Material e Métodos}

A área experimental localiza-se no Instituto Federal de Educação, Ciência e Tecnologia do Triângulo Mineiro, Campus Uberaba (IFET), no município de Uberaba, MG, (19³9’19’'S, 47 $57^{\prime} 27^{\prime \prime} W$, a cerca de $795 \mathrm{~m}$ de altitude). 
A precipitação média anual é de $1.600 \mathrm{~mm}$; a temperatura média anual é de $22,6{ }^{\circ} \mathrm{C}$ e a umidade relativa do ar média é de $68 \%$. O clima é classificado como Aw, tropical quente, segundo a classificação de Köppen, apresentando inverno frio e seco. O solo da área experimental foi classificado como Latossolo Vermelho Distrófico (EMBRAPA, 2006). Apresenta as seguintes características na camada arável (0-20 $\mathrm{cm}) ; 180 \mathrm{~g} \mathrm{~kg}^{-1}$ de argila, $\mathrm{pH} \mathrm{H}_{2} \mathrm{O}(1: 2,5) 6,3 ; 17 \mathrm{mg}$

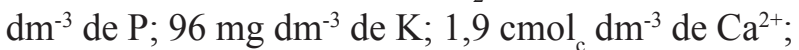
$0,6 \mathrm{cmol}_{\mathrm{c}} \mathrm{dm}^{-3}$ de $\mathrm{Mg}^{2+} ; 2,0 \mathrm{cmol}_{\mathrm{c}} \mathrm{dm}^{-3}$ de $\mathrm{H}+\mathrm{Al} \mathrm{e}$ $16 \mathrm{~g} \mathrm{dm}^{-3}$ de matéria orgânica. As análises foram realizadas segundo Embrapa (1997).

O experimento foi instalado em agosto de 2000, com o plantio das seguintes espécies como plantas de cobertura: milheto (Pennisetum americanum sin. tiphoydes), braquiária (Brachiaria brizantha), crotalária juncea (Crotalarea juncea), além de ser também utilizada uma área em pousio e uma área testemunha (preparo convencional, onde são feitas as práticas de aração e gradagem). $\mathrm{Na}$ área de pousio foi feita a identificação das plantas invasoras, sendo constatada a presença de espécies de diversas famílias com predomínio de gramíneas (em maior proporção), além de Solanaceae, Compositae, Portulacaceae, Cyperaceae, Rubiaceae e Amaranthaceae.

Aproximadamente 110 dias após o plantio, as plantas de cobertura foram dessecadas aplicandose $1.440 \mathrm{~g} \mathrm{ha}^{-1}$ de glifosato. Quinze dias após dessecação, as parcelas foram subdivididas em duas áreas de $20 \mathrm{~m}^{2}$, e em novembro de 2000 plantou-se milho híbrido duplo AG 1051 e soja MG/BR-46, sendo feito a inoculação das sementes de soja com Bradyrhizobium japonicum. Durante a condução do experimento fez o controle de pragas, doenças e plantas daninhas necessários para o ciclo das culturas. Logo após a colheita do milho e da soja, foi realizado novamente o plantio das coberturas vegetais, nos mesmos locais, e posteriormente um novo plantio das culturas. Este ciclo foi repetido anualmente até 2007. Uma área de Cerrado preservado foi utilizada como referência da comunidade indígena de FMAs do solo.
O delineamento experimental adotado foi de blocos casualizados, com 4 repetições, em um esquema de parcelas subdivididas, sendo as parcelas constituídas pelos cinco sistemas de manejo do solo avaliados - pousio, preparo convencional e plantio direto com uso dos cultivos de cobertura de crotalária, milheto e braquiária -, e as subparcelas pelos cultivos de soja e milho. Cada parcela experimental tinha a dimensão de $4 \mathrm{~m}$ x $10 \mathrm{~m}\left(40 \mathrm{~m}^{2}\right)$, num total de 20 parcelas ( 5 sistemas de manejo e 4 repetições). E, cada subparcela apresentava $20 \mathrm{~m}^{2}$, sendo $20 \mathrm{~m}^{2}$ com soja e $20 \mathrm{~m}^{2}$ com milho, totalizando $40 \mathrm{~m}^{2}$ de área total por parcela.

Em fevereiro de 2007, para as avaliações da colonização micorrízica e densidade de esporos de FMAs, foram coletados raiz e solo das culturas de milho e soja em cada subparcela, e também em área de Cerrado, nas profundidades de 0,0-0,05 $\mathrm{m}$ e 0,05-0,10 $\mathrm{m}$, área de ocorrência das raízes metabolicamente ativas destas culturas. As raízes frescas foram lavadas em água corrente, clarificadas e coloridas segundo Koske e Gemma (1989) e Grace e Stribley (1991). Em seguida, foram levadas ao microscópio óptico num aumento de 200x para serem avaliadas quanto à colonização radicular, conforme McGonigle e Fitter (1990). Para a contagem do número de esporos de FMAs, amostras de $50 \mathrm{~g}$ de solo foram processadas, segundo uma associação dos métodos de decantação e peneiramento úmido (GERDEMANN; NICOLSON, 1963) e de centrifugação e flutuação em sacarose (JENKINS, 1964). A quantificação foi realizada em placas de acrílico com anéis concêntricos, sob microscópio estereoscópico (40x).

Para a avaliação da diversidade de FMAs foi coletado o solo na área de Cerrado e nas subparcelas na profundidade de 0,0 a $0,10 \mathrm{~m}$. A identificação das espécies foi feita conforme Schenk e Perez (1987) e Morton e Benny (1990), sob microscópio óptico, após a fixação dos esporos em PVLG ou PVLG mais reagente de Melzer.

Para análise estatística dos dados, foi feita a avaliação da normalidade dos dados (teste de 
Lilliefors), homogeneidade das variâncias dos erros (teste de Cochran e Barttlet) utilizando o programa estatístico SAEG versão 5.0 (Sistema de Análises Estatísticas e Genéticas - Universidade Federal de Viçosa). A porcentagem de colonização micorrízica apresentou os pré-requisitos da estatística paramétrica, enquanto o número de esporos no solo foi transformado e destransformado por logatitmo neperiano $\left(\log _{\mathrm{e}} \mathrm{n}=\mathrm{y}\right)$ para obter a normalidade e homogeneidade de variância dos erros. Posteriormente, os resultados foram submetidos à análise de variância com aplicação do Teste F e os valores médios comparados entre si pelo teste de Tukey a $5 \%$, utilizando-se o sistema computacional SAEG .

Também se realizou análise de componentes principais (ACP) por meio do aplicativo SAS (SAS, 1999), para a camada de 0,0-10 cm, para a diversidade de FMAs. Outra ACP foi feita para avaliar o comportamento das áreas cultivadas comparadas com a área de Cerrado, englobando algumas características físicas e químicas do solo das áreas avaliadas que se encontram descritas, com as respectivas metodologias, em Pereira et al. (2010; 2012), tais como carbono orgânico total (COT), carbono das substâncias húmicas (fração acido fúlvico -C-FAF, fração ácido húmico - C-FAH, fração humina - C-HUM), carbono das frações granulométricas (carbono orgânico particulado COp, carbono orgânico associado aos minerais COam), matéria orgânica leve (MOL), densidade do solo (Ds), fósforo remanescente (Prem) e as variáveis de FMAs (Colonização micorrízica COL - e número de esporos - ESP).

\section{Resultados e Discussão}

Verificou-se efeito da cultura sobre a porcentagem de colonização micorrízica e para o número de esporos dos FMAs na rizosfera das plantas nas duas profundidades estudadas. Porém, efeito significativo foi observado apenas para as coberturas e sua interação com culturas (culturas $\mathrm{x}$ coberturas) na profundidade $0,0-0,05 \mathrm{~m}$ (Tabela 1 ).

Tabela 1. Resumo das análises de variância apresentando os valores de F calculados para as causas de variação e os coeficientes de variação.

\begin{tabular}{|c|c|c|c|c|}
\hline \multirow[b]{2}{*}{ Causas de variação } & \multicolumn{4}{|c|}{ Variáveis } \\
\hline & $\begin{array}{c}\text { Colonização } \\
0,0-0,05 m\end{array}$ & $\begin{array}{l}\text { Colonização } \\
0,05-0,10 \mathrm{~m}\end{array}$ & $\begin{array}{l}\mathrm{n}^{\circ} \text { de esporos } \\
0,0-0,05 m^{(1)}\end{array}$ & $\begin{array}{c}\mathrm{n}^{\circ} \text { de esporos } \\
0,05-0,10 \mathrm{~m}^{(1)}\end{array}$ \\
\hline Culturas & $38,85^{* *}$ & $61,72^{* *}$ & $175,89^{* *}$ & $25,26^{* *}$ \\
\hline Cobertura Vegetal & $10,27^{* *}$ & 2,62 ns & $0,0326^{* *}$ & $1,20^{\text {ns }}$ \\
\hline Culturas x Cobertura Vegetal & $4,14^{* *}$ & $1,06^{\mathrm{ns}}$ & $0,0146^{* *}$ & 0,63 ns \\
\hline C.V erro $1(\%)$ & 3,89 & 3,69 & 1,70 & 3,88 \\
\hline C.V. erro $2(\%)$ & 3,25 & 5,25 & 3,84 & 5,03 \\
\hline
\end{tabular}

${ }^{* *}$ :significativo a $5 \%$; ${ }^{\text {ns: }}$ não significativo; C.V.=coeficiente de variação.

(1) Número de esporos foram transformados e destransformados por logaritmo neperiano para obter normalidade e homogeneidade de variância dos dados.

Fonte: Elaboração dos autores.

A colonização micorrízica foi alta para ambas as culturas, sendo superior a $90 \%$ na área com milho e $80 \%$ na área com soja, destacando-se que na área com a cultura do milho verificaram-se maiores valores de colonização micorrízica das raízes, independente da cobertura avaliada, com exceção à crotalária (Tabela 2). Resultados semelhantes foram encontrados por Faria et al. (2009), que verificaram colonização micorrízica superior a $70 \%$ na cultura do milho, sobre milheto, bem como menor colonização micorrízica na cultura da soja sobre milheto, com $23 \%$. 
Tabela 2. Colonização micorrízica e número de esporos $\left(50 \mathrm{~g}\right.$ de solo $\left.\mathrm{seco}^{-1}\right)$ de milho e soja em plantio direto em solo de Cerrado, na profundidade de 0,0-0,05 m,.

\begin{tabular}{lccccc}
\hline \multicolumn{5}{c}{ Sistemas de manejo / Variavel avaliada } \\
\hline \multirow{2}{*}{ Tratamentos } & \multicolumn{5}{c}{ Colonização Micorrízica (\%) } \\
\cline { 2 - 5 } & Braquiária & Milheto & Crotalária & Convencional & Pousio \\
\hline \multirow{2}{*}{ Milho } & $99,5 \mathrm{aA}$ & $98,7 \mathrm{aA}$ & $95,5 \mathrm{aA}$ & $95,2 \mathrm{aA}$ & $94,5 \mathrm{aA}$ \\
Soja & $93,0 \mathrm{bA}$ & $91,5 \mathrm{bA}$ & $94,0 \mathrm{aA}$ & $89,0 \mathrm{bA}$ & $80,7 \mathrm{bB}$ \\
\hline \multicolumn{5}{c}{ Milho } & \multicolumn{5}{c}{ Número de Esporos (n ${ }^{\mathbf{0}}$ por 50 g de solo) } \\
Soja & $411 \mathrm{aB}$ & $608 \mathrm{aA}$ & $414 \mathrm{aB}$ & $301 \mathrm{aC}$ & $306 \mathrm{aC}$ \\
\hline
\end{tabular}

Médias seguidas de mesma letra, minúscula na coluna compara os tratamentos para cada sistema de manejo e maiúscula na linha compara os sistemas de manejo para cada tratamento, não diferem entre si pelo teste de Tukey a 5\% de probabilidade.

Fonte: Elaboração dos autores.

Para a cultura do milho não se observou diferenças entre as coberturas e manejo na colonização micorrízica. $\mathrm{Na}$ cultura da soja, a área em pousio apresentou menor colonização micorrízica nas plantas espontâneas que nas raízes de soja das demais áreas. É complexo comparar a colonização micorrízica de diferentes plantas devido à compatibilidade diferenciada com as espécies de FMAs existentes no solo e à variação nas características genéticas das plantas (SMITH; GIANINAZZI-PEARSON, 1988). Em função deste padrão, a menor colonização micorrízica na área de pousio com soja pode ser decorrente da presença de plantas da família Cyperaceae, sendo esta uma das poucas plantas fanerogâmicas que não apresentam associação micorrízica, sem a presença de tipos morfológicos como arbúsculo e enovelados de hifas (SOUZA et al., 2006). Geralmente, em áreas de pousio, ocorrem espécies antagônicas capazes de selecionar fungos micorrízicos sensíveis à supressão microbiana do solo (KITT; DANIELS-HETRICK; WILSON, 1987).

A colonização micorrízica é uma característica que pode ser afetada por inúmeros fatores como a espécie vegetal, a idade da planta, a densidade de raízes, dos propágulos de FMAs no solo, a eficiência de colonização de FMAs e o manejo do solo (AFEK et al., 1990). Dessa forma, a maior colonização e número de esporos na área com milho
(Tabela 2 e Figura 1) devem-se, provavelmente, à maior eficiência fotossintética das gramíneas, que podem investir fotossintatos na simbiose com os FMAs, mantendo níveis mais altos de colonização, para obter retorno com a maior absorção de $\mathrm{P}$ (CORDEIRO et al., 2005).

A maior esporulação é atribuída às plantas com sistema radicular abundante e de rápido crescimento, com melhor contato entre raízes e propágulos de FMAs e grande capacidade de fornecer fotossintatos ao fungo, tal como é o sistema radicular das gramíneas (DANIELSHETRICK; BLOOM, 1986). Além destes fatores, a cultura do milho proporciona aumentos na colonização micorrízica e na densidade de esporos de fungos micorrízicos, devido à exsudação de compostos bioativos que estimulam a germinação e o crescimento micelial (SIQUEIRA; KLAUBERGFILHO, 2000). Portanto, um manejo do solo que utilize a cultura do milho proporciona aumentos na colonização micorrízica das raízes (CORDEIRO et al., 2005; FARIA et al., 2009; CAMPOS et al., 2010).

Quanto ao número de esporos verificou-se que a cultura do milho também promoveu maior esporulação na rizosfera do que a cultura da soja, exceto para as áreas com plantio convencional e pousio, onde não houve diferenças entre as culturas (Tabela 2). As coberturas vegetais e o manejo 
influenciaram na densidade de esporos, sendo que na área de milho, a cobertura de milheto promoveu maior esporulação, seguido das coberturas de braquiária e crotalária. As áreas com cultivo convencional e em pousio apresentaram menor densidade de esporos. $\mathrm{Na}$ área de soja não houve diferenças entre os tratamentos de cobertura e manejo. O preparo do solo convencional (aração e gradagem) pode romper a rede de hifas e reduzir o potencial de inóculo de FMAs, expondo seus propágulos, como hifas, esporos e raízes colonizadas a altas temperaturas, excesso de oxigênio (oxidação) e a predadores, tornando-os inviáveis (KABIR, 2005). Isto pode ter levado nas áreas cultivadas com milho, a de cultivo convencional ter menor densidade de esporos que nas de plantio direto.

$\mathrm{Na}$ literatura é comum encontrar trabalhos que relatem aumento tanto na colonização radicular quanto no número de esporos de FMAs no sistema plantio direto em relação ao convencional (SILVEIRA; FREITAS, 2007; SCHNEIDER et al., 2011) ou em sistemas em que o solo seja menos perturbado (MCGONIGLE; MILLER, 1996; SCHNEIDER et al., 2011). Todavia, isso parece não ser uma regra e depende muito da planta hospedeira, das espécies de fungos micorrízicos predominantes e da especificidade (SILVEIRA; FREITAS, 2007). Isso pode ser observado no trabalho de Fernandes (1995), onde foram encontrados valores maiores de colonização de raízes da aveia em plantio direto que no convencional; porém, o autor verificou que na soja a colonização foi semelhante entre os sistemas.

$\mathrm{Na}$ profundidade de 0,05-0,10 $\mathrm{m}$ houve apenas efeito das diferentes culturas sobre essas variáveis, sendo que a cultura do milho apresentou maior colonização micorrízica e esporularação na rizosfera que a cultura da soja (Figura 1). Provavelmente, os efeitos dos diferentes sistemas radiculares, apresentados por culturas e coberturas vegetais, se concentraram na camada superficial do solo (0-5 $\mathrm{cm})$, favorecendo o contato entre raízes e propágulos de FMAs, com grande capacidade de fornecimento de fotossintatos aos fungos por meio dos diferentes sistemas radiculares (DANIELS-HETRICK; BLOOM, 1986), o que acaba por favorecer a maior esporulação e colonização na camada superficial quando comparada com a camada de $5-10 \mathrm{~cm}$.

Figura 1. Porcentagem de colonização micorrízica (A) e densidade de esporos na rizosfera (B) de milho e soja na profundidade de 0,05- 0,10 m em solo de Cerrado.
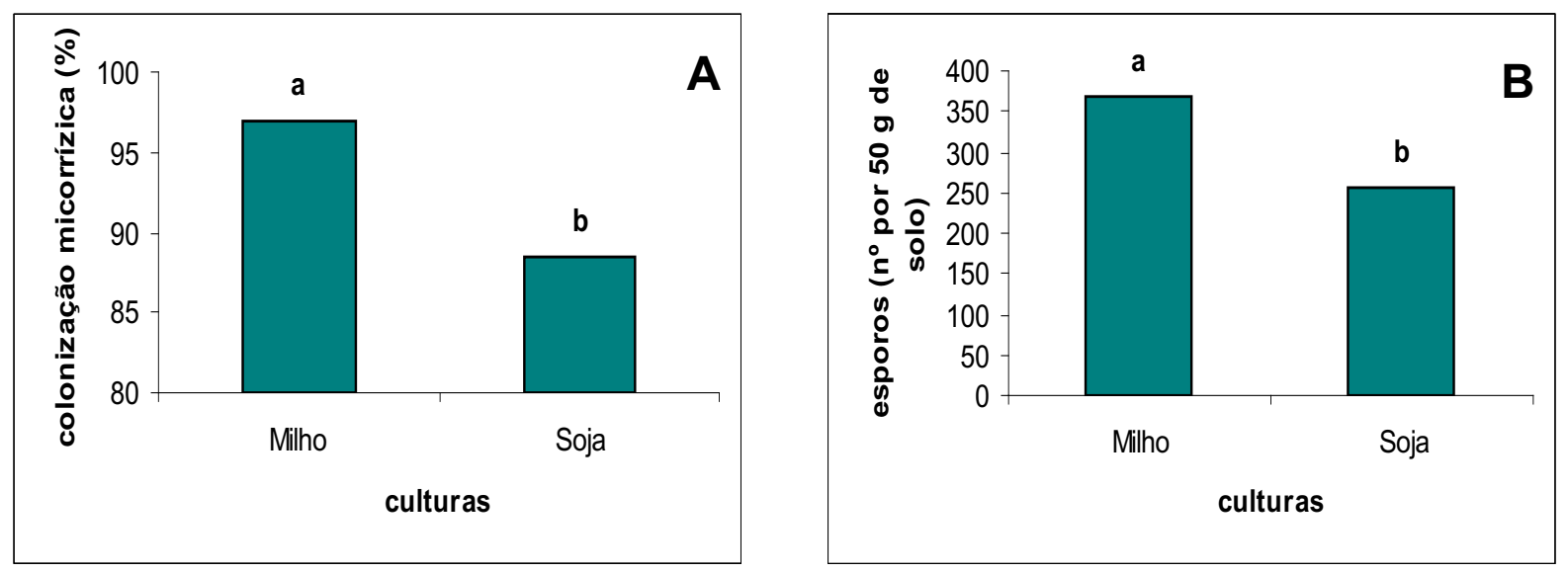

Fonte: Elaboração dos autores. 
A cultura do milho plantada sobre milheto foi o tratamento com maior número de esporos, provavelmente em função da eficiência fotossintética destes dois hospedeiros (Tabela 2). Assim, as coberturas vegetais dentro do sistema de plantio direto, além de contribuírem positivamente nas propriedades físicas e químicas do solo (PEREIRA et al., 2010), destacam-se também pela alteração das propriedades biológicas do solo. Em específico para fungos micorrízicos, as coberturas vegetais exercem o papel de multiplicadoras de esporos de FMAs, garantindo o elevado potencial de inóculo nestes solos (CARNEIRO et al., 1995). Entretanto, a escolha da espécie vegetal para cobertura a ser utilizada é importante, pois estas podem promover alterações quantitativas e qualitativas na população de FMAs autóctones, pois a associação micorrízica é favorecida pela existência de exsudatos radiculares que contém moléculas que estimulam a germinação de esporos e o crescimento de fungos micorrízicos (BÉCARD; PICHÉ, 1989).

$\mathrm{O}$ mecanismo de reconhecimento entre as bactérias e fungos e a planta obedece a um complexo conjunto de informações entre eles, que inicia com a quimiotaxia, ou seja, uma atração química entre os indivíduos por meio de exsudatos radiculares. As condições do meio também podem interferir na esporulação. Segundo Faria et al. (2009), em trabalho desenvolvido com culturas de milho e soja cultivadas sobre a palhada de milheto e soja, o autor observou que a esporulação e número de esporos da cultura da soja foi menor sobre as palhadas de milheto e soja quando comparada com a cultura do milho sobre as mesmas plantas de cobertura. Segundo Benedetti et al. (2005), as leguminosas são capazes de produzir uma variabilidade de metabólicos, como flavonóides, que exercem efeitos diferenciados sobre os fungos micorrízicos. Neste estudo, de acordo com Benedetti et al. (2005) e Faria et al. (2009), nas áreas com a soja, pode ter ocorrido a liberação de exsudatos radiculares que contém moléculas que proporcionam menor estímulos a germinação de esporos e o crescimento de FMAs quando comparado com as áreas com milho.

Outra atividade propiciada pela cultura do milho em relação à comunidade microbiana é o estimulo a emergência e crescimento micelial de FMAs, devido a exsudação de compostos secundários bioativos que interagem com a comunidade microbiana promovendo o incremento destas variáveis (SIQUEIRA; KLAUBERG-FILHO, 2000), fato importante na evidência de uma alelopatia positiva. Portanto, neste caso um manejo do solo que utilize a cultura do milho proporciona aumentos na colonização micorrízica das raízes, conforme já relatado por Faria et al. (2009).

Em relação à diversidade de espécies verificouse que a composição da comunidade de FMAs foi diferente para os tratamentos de manejos agrícolas, sendo encontradas 18 espécies de FMAs, destacando-se os gêneros Acaulospora, Gigaspora, Scutellospora e Glomus (Tabela 3, Figuras 2A).

A área de Cerrado apresentou 10 espécies do total encontrado, sendo Acaulospora. foveata e Scutellospora reticulata encontradas apenas nesta área (Tabela 3, Figura 2A). Isto indica que estas espécies são indicadoras de ambiente sem interferência antrópica e segundo Stürmer e Siqueira (2005), que classificam as espécies de FMAs de acordo com seu grau de ocorrência no ambiente em generalistas, intermediárias e raras, a espécie Scutellospora reticulata esta entre as raras. 
Tabela 3. Espécies de FMAS encontradas nas áreas de milho e soja em plantio direto em solo de Cerrado, na profundidade de $0,0-0,10 \mathrm{~m}$.

\begin{tabular}{|c|c|c|c|c|c|c|c|}
\hline \multirow{2}{*}{ Espécies } & \multicolumn{7}{|c|}{ Sistemas de manejo / Cultura avaliada } \\
\hline & & Braquiária & Milheto & Crotalária & Convenc. & Pousio & Cerrado \\
\hline \multirow{2}{*}{ Ac. scrobiculata } & Milho & $\mathrm{x}$ & $\mathrm{x}$ & $\mathrm{x}$ & - & $\mathrm{x}$ & \multirow{2}{*}{$\mathrm{x}$} \\
\hline & Soja & $\mathrm{x}$ & $\mathrm{x}$ & $\mathrm{x}$ & $\mathrm{x}$ & $\mathrm{x}$ & \\
\hline \multirow{2}{*}{ Ac. foveata } & Milho & - & - & - & - & - & \multirow{2}{*}{$\mathrm{x}$} \\
\hline & Soja & - & - & - & - & - & \\
\hline \multirow{2}{*}{ Ac. scavata } & Milho & $\mathrm{x}$ & - & - & - & - & \multirow{2}{*}{-} \\
\hline & Soja & - & $\mathrm{x}$ & - & - & $\mathrm{x}$ & \\
\hline \multirow{2}{*}{ Ac. mellea } & Milho & $\mathrm{x}$ & - & $\mathrm{x}$ & - & & \multirow{2}{*}{$\mathrm{x}$} \\
\hline & Soja & - & $\mathrm{x}$ & - & - & $\mathrm{x}$ & \\
\hline \multirow{2}{*}{ Acaulospora sp } & Milho & - & - & $\mathrm{x}$ & - & - & \multirow{2}{*}{$\mathrm{x}$} \\
\hline & Soja & - & - & $\mathrm{x}$ & - & - & \\
\hline \multirow{2}{*}{ Ar. leptoticha } & Milho & $\mathrm{x}$ & $\mathrm{x}$ & - & - & - & \multirow{2}{*}{$\mathrm{x}$} \\
\hline & Soja & $\mathrm{x}$ & $\mathrm{x}$ & - & - & - & \\
\hline \multirow{2}{*}{ Gigaspora sp } & Milho & - & - & $\mathrm{x}$ & $\mathrm{x}$ & $\mathrm{x}$ & \multirow{2}{*}{-} \\
\hline & Soja & $\mathrm{x}$ & $\mathrm{x}$ & - & - & - & \\
\hline \multirow{2}{*}{ Gl. formosanum } & Milho & - & - & - & $\mathrm{x}$ & - & \multirow{2}{*}{$\mathrm{x}$} \\
\hline & Soja & - & - & - & $\mathrm{x}$ & - & \\
\hline \multirow{2}{*}{ Gl. microagregatum } & Milho & - & - & - & - & - & \multirow[b]{2}{*}{ - } \\
\hline & Soja & - & - & - & - & $\mathrm{x}$ & \\
\hline \multirow{2}{*}{ Gl. macrocarpum } & Milho & - & $\mathrm{x}$ & - & - & - & \multirow{2}{*}{$\mathrm{x}$} \\
\hline & Soja & $\mathrm{x}$ & $\mathrm{x}$ & - & $\mathrm{x}$ & - & \\
\hline \multirow{2}{*}{ Gl. tortuosum } & Milho & $\mathrm{x}$ & - & $\mathrm{x}$ & - & $\mathrm{x}$ & \multirow[b]{2}{*}{$\mathrm{x}$} \\
\hline & Soja & $\mathrm{x}$ & $\mathrm{x}$ & $\mathrm{x}$ & $\mathrm{x}$ & $\mathrm{x}$ & \\
\hline \multirow{2}{*}{ Gl. clarum } & Milho & $\mathrm{x}$ & - & - & - & - & \multirow{2}{*}{-} \\
\hline & Soja & - & - & - & - & - & \\
\hline & Milho & - & - & - & - & - & \\
\hline Glomus sp & Soja & - & - & - & - & $\mathrm{x}$ & - \\
\hline & Milho & $\mathrm{x}$ & $\mathrm{x}$ & $\mathrm{x}$ & $\mathrm{x}$ & $\mathrm{x}$ & 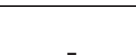 \\
\hline Sc. scutata & Soja & $\mathrm{x}$ & $\mathrm{x}$ & $\mathrm{x}$ & $\mathrm{x}$ & $\mathrm{x}$ & - \\
\hline & Milho & - & $\mathrm{x}$ & $\mathrm{x}$ & $\mathrm{x}$ & $\mathrm{x}$ & \\
\hline Sc. gregaria & Soja & - & $\mathrm{x}$ & $\mathrm{x}$ & $\mathrm{x}$ & - & - \\
\hline & Milho & $\mathrm{x}$ & - & - & - & - & \\
\hline Sc. heterogama & Soja & - & - & - & - & - & $\mathrm{x}$ \\
\hline & Milho & - & - & - & - & - & \\
\hline Sc. reticulata & Soja & - & - & - & - & - & $\mathrm{x}$ \\
\hline & Milho & $\mathrm{x}$ & - & - & - & - & \\
\hline Sclerocystis sp & Soja & $\mathrm{x}$ & - & - & - & - & - \\
\hline
\end{tabular}

Ac=Acaulospora; Ar= Archaeospora; $G l=$ Glomus; $S c=$ Scutellospora . Convenc $=$ Preparo convencional do solo

Fonte: Elaboração dos autores. 
Figura 2. Análise de componentes principais afetando a composição de espécies das comunidades de FMAs nas áreas de estudo (Figura 2A) e análise de agrupamento das diferentes áreas do estudo quanto a presença e ausência de espécies de FMAs (Figura 2B).
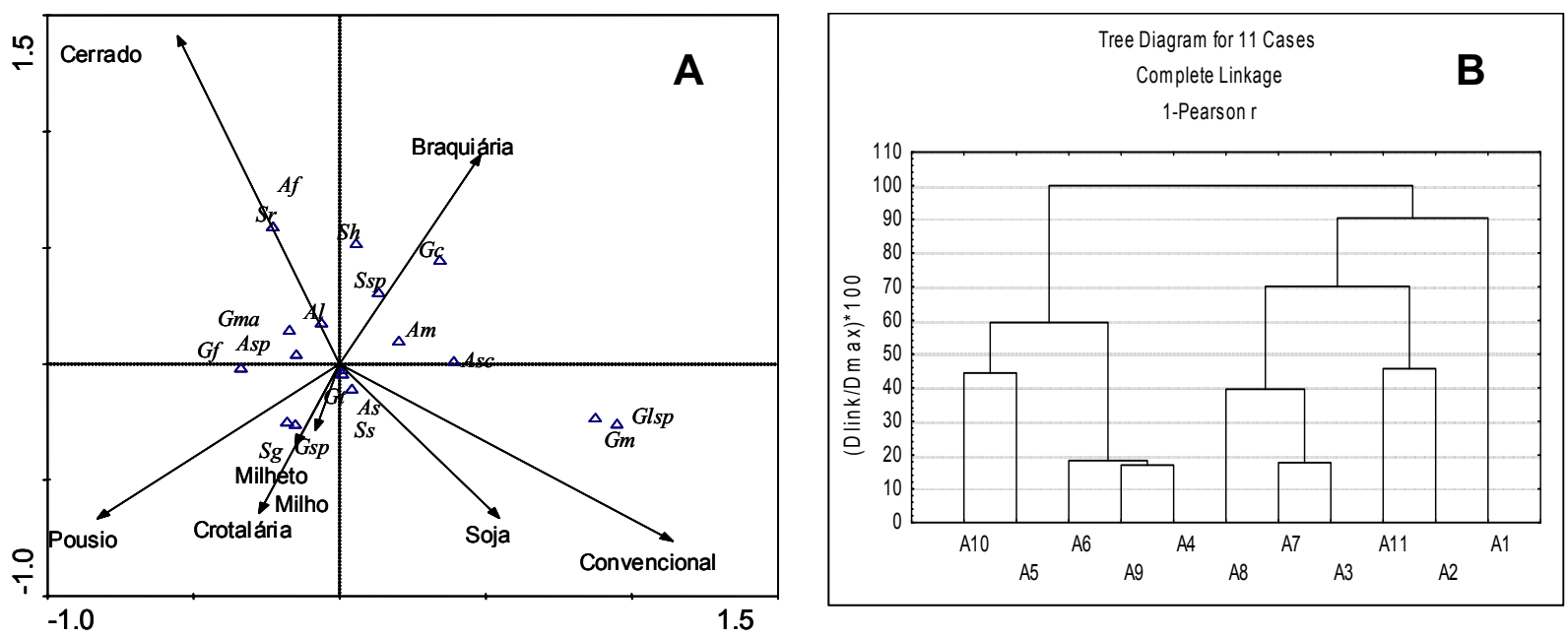

Legendas da Figura 2A: As: Acaulospora scrobiculata; Af: Acaulospora foveata; Asc: Acaulospora scavata; Am: Acaulospora mellea; Asp: Acaulospora sp; Al: Archaeospora leptoticha; Gsp: Gigaspora sp; Gf: Glomus formosanum; Gm: Glomus microagregatum; Gma: Glomus macrocarpum; Gt: Glomus tortuosum; Gc: Glomus clarum; Glsp: Glomus sp; Ss: Scutellospora scutata; Sg: Scutellospora gregária; Sh: Scutellospora heterogama; Sr: Scutellospora reticulata; Ssp: Sclerocystis Sp. Legendas da Figura 2B: A1: Cerrado; A2: Milho-braquiária; A3: Milho-milheto; A4: Milho-crotalaria; A5: Milho-convencional; A6: Milho-pousio; A7: Soja-braquiária; A8: Soja-milheto; A9: Soja-crotalária; A10:Soja-convencional; A11: Soja-pousio.

Fonte: Elaboração dos autores.

A Figura 2A mostra que há um grande distanciamento dos tratamentos manejados sob plantio direto e convencional da área de Cerrado natural, em função das espécies deFMAs encontradas nas áreas (Tabela 3). O plantio convencional foi o manejo que mais se distanciou da área de Cerrado e dos demais sistemas de manejo avaliados, tendo apresentado o menor número de espécies de FMAs em relação as culturas de milho e soja (Tabela 3). As culturas da soja e do milho diferem do Cerrado natural, mas induzem a comunidades de FMAs com tendências diferentes entre si na composição específica.(Tabela 3). Quanto às coberturas vegetais, milheto, crotalária e a vegetação espontânea do pousio induzem a composição da comunidade de FMAs semelhante, mas oposta a composição da comunidade induzida pela braquiária. Este padrão se deve a presença de três espécies de FMAs (Glomus clarum, Scutellospora heterogama e Sclerocystis $\mathrm{sp)}$ que foram encontradas apenas na cobertura de braquiária, com destaque para a interação gramínea x gramínea (milho sobre braquiária), que apresentaram as três espécies, sendo na área com soja verificada apenas Sclerocystis sp (Tabela 3).

A análise de agrupamento das áreas quanto à presença e ausência de espécies de FMAs (Figura 2B) mostra a separação das áreas em três principais grupos. Um grupo formado pelo Cerrado nativo (A1) que se distanciou dos outros dois grupos em pelo menos 90\% de distância de ligação. Um segundo grupo formado pelos tratamentos que envolvem principalmente as coberturas com braquiária e milheto: Milho-braquiária (A2); Milhomilheto (A3); Soja-braquiária (A7); Soja-milheto (A8) e Soja-pousio (A11). E um terceiro grupo formado pelos tratamentos: Soja-convencional (A10), Milho-convencional (A5), Milho-pousio (A6), Soja-crotalária (A9) e Milho-crotalária (A4) que se distanciaram ao máximo do Cerrado. 
Este agrupamento é devido às espécies encontradas nessas áreas, sendo no Cerrado verificado o maior número de espécies (10), seguida pelas áreas de braquiária (10) e milheto (9) e em menor numero, nas áreas de preparo convencional (7), pousio (8) e crotalária (7) (Tabea 3). Nas condições dos ecossistemas naturais, onde a ação antrópica é dada como nula ou mínima, espera-se que a diversidade de fungos micorrízicos seja maior e mais estabilizada do que em sistemas agrícolas convencionais (CORDEIRO et al., 2005). Em geral, a comunidade dos FMAs é numerosa em sistemas agrícolas que englobam, principalmente, uso reduzido de agroquímicos, cultivo mínimo, rotação de culturas e plantio de gramíneas (BENEDETTI et al., 2005). Este padrão pode ser devido à combinação das práticas citadas anteriormente com plantas com alto grau de micotrofia empregadas em cobertura, tais como as gramíneas, contribuindo para manter elevada densidade de hifas, elevado potencial de inóculo, estabilização do solo e, assim, manter a sustentabilidade da produção (KABIR, 2005). Em contra-partida, no preparo convencional do solo, o uso de revolvimento intensivo do solo afeta negativamente os FMAs, e assim contribui para o menor número de espécies encontradas, reduzindo a sustentabilidade deste sistema quando comparado ao sistema plantio direto (ALGUACIL et al., 2008; SCHNEIDER et al., 2011).

$\mathrm{Na}$ ACP englobando algumas características do solo e as variáveis de FMAs (colonização e $\mathrm{n}^{\mathrm{o}}$ de esporos) juntamente com área testemunha (Cerrado), na camada de 0,0-0,10 m, verificouse que a distribuição das variáveis selecionadas apresentou variância acumulada de 75,36 \% para os eixos F1 e F2, sendo que o eixo F1 foi capaz de explicar $61,71 \%$ e o F2, 13,65 \% dessa variância (Figura 3).
Os componentes principais, dispostos num espaço de duas dimensões (F1 e F2), representam variabilidade suficiente para indicar algum padrão a ser interpretado (GOMES et al., 2004), sendo as variáveis analisadas representadas por setas, que indicam a direção do gradiente máximo, em que o comprimento da seta é proporcional à correlação da característica com os eixos e à sua importância na explicação da variância projetada em cada eixo (LOSS et al., 2009b). Neste sentido, destacase para correlação com o eixo $\mathrm{F} 1$, as variaveis COT, COam, COL e C-FAF, que apresentaram autovetores positivos, e C-HUM, C-FAH e COp, que apresentaram autovetores negativos. Também se observa a influência do Prem, Ds e MOL, no eixo F1, com autovetores negativos. No eixo F2, destaca-se apenas a variável ESP apresentando autovetor positivo e por isso, somente $12,55 \%$ da variabilidade é explicada pelo eixo F2 (Figura 1).

Observou-se a formação de três grupos, sendo a área de Cerrado a mais distante das áreas cultivadas com milho e soja e, entre essas, verifica-se que, independente das coberturas vegetais, as culturas se distanciam, mostrando a baixa similaridade entre elas, sendo a área com soja na parte inferior do gráfico e a área com milho na parte superior (Figura 3). Este padrão evidencia que na área de milho, a componente biológica (COL e ESP) é mais expressiva (com maiores valores, Tabela 2, e maior numero de espécies de FMAs, Tabela 3) quando comparada com a cultura da soja. Este resultado é semelhante ao observado na Figura 2, onde a ACP da diversidade de FMAs conseguiu separar a área de Cerrado (10 espécies de FMAs) das áreas das culturas e dessas, cada uma ficou em um quadrante do gráfico, sendo verificado maior presença de espécies de FMAs na área de milho quando comparada com a área com soja (Tabela 3). 
Figura 3. Diagrama de ordenação produzido por análise de componentes principais na profundidade de $0-10 \mathrm{~cm}$ das características físicas, químicas e biológicas do solo, em que: $\mathrm{M}$ - milho; $\mathrm{S}$ - soja; 1 - milheto; 2 - crotalária; 3 braquiária; 4 - plantio convencional; 5 - pousio; 6 - Cerrado.

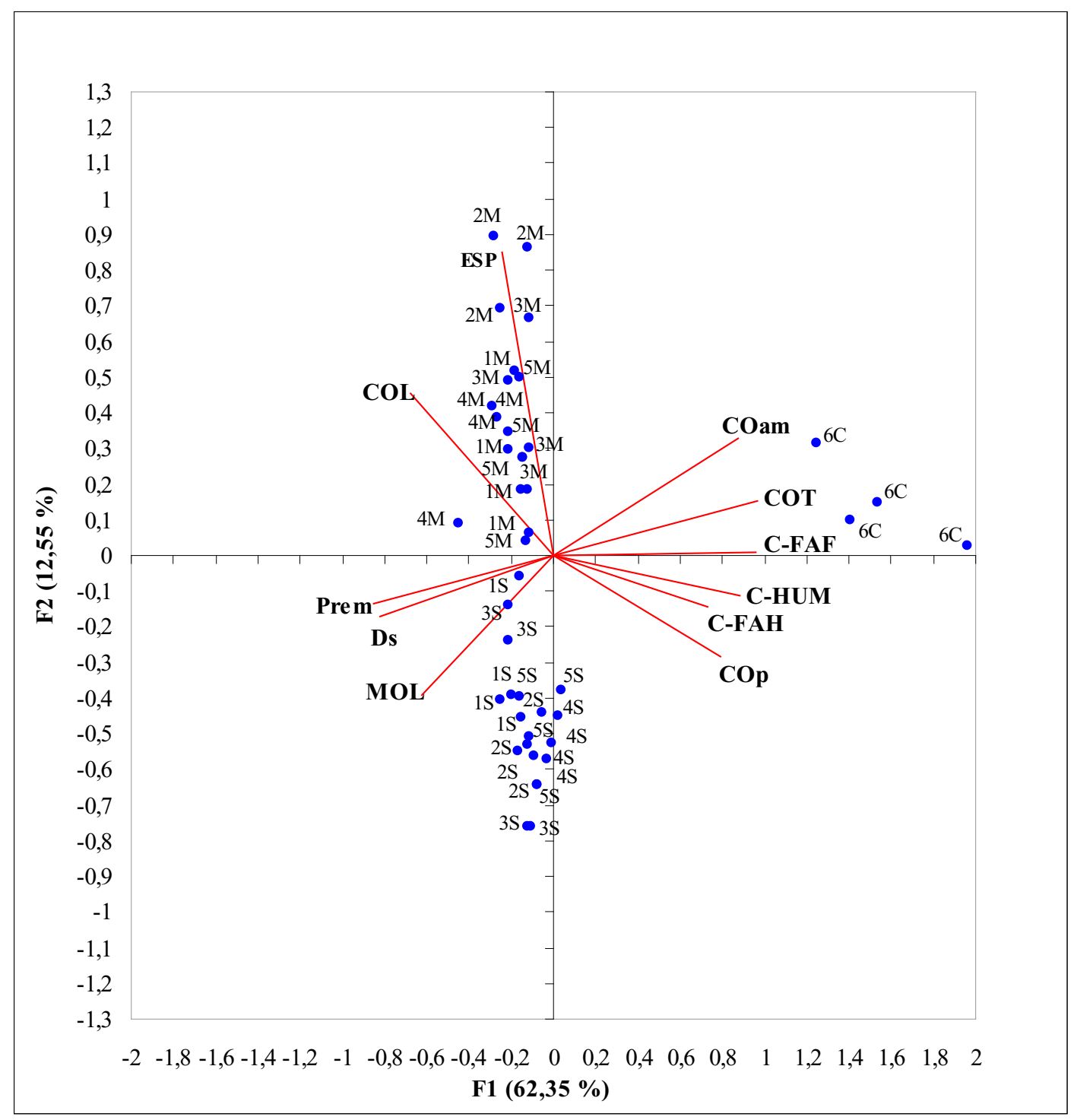

Fonte: Elaboração dos autores.

$\mathrm{Na}$ Figura 3, os três grupos formados são separados por variáveis diferentes: na área de Cerrado, têm-se as variáveis COT, carbono das frações granulométricas e das substâncias húmicas, na área com a cultura da soja verifica-se as variáveis Prem, Ds e MOL, e na área de cultivo de milho, as variáveis colonização (COL) e número de esporos (ESP). A área de Cerrado, por não ter influência antrópica, apresenta uma combinação de aporte de material vegetal e menor taxa de decomposição do mesmo. Dessa forma tem maiores teores de matéria orgânica na camada de 0,0-10,0 cm, conseqüentemente, de carbono e das frações que o compõem, como pode ser evidenciado na Figura 3.

Entre as áreas de cultivo, verificou-se que na cultura de milho, as variáveis biológicas são preponderantes, o que pode estar relacionado com a maior eficiência fotossintética das plantas de milho 
(gramíneas) e estabelecimento de simbiose com os FMAs, sendo verificado maior número de espécies de FMAs na área de milho (Tabela 3). Já na área com soja as variáveis MOL, Ds e o Prem (PEREIRA et al., 2010) podem estar relacionadas com a menor taxa de decomposição dos restos culturas da soja quando comparado com plantas de milho.

De maneira geral, pode-se concluir que os dados referentes aos fungos micorrízicos, colonização micorrízica e número de esporos, foram mais eficientes para evidenciar diferenças entre áreas de cultivo em sistema plantio direto e diferentes coberturas vegetais sob solo de Cerrado do que as outras variáveis relacionadas às frações da MOS, para as condições do presente estudo.

\section{Conclusões}

A cultura do milho, quando comparada com a da soja, apresenta maior colonização micorrízica para os sistemas com braquiária, milheto, convencional e pousio, e densidade de esporos para os sistemas com braquiária, milheto e crotalária. O manejo do solo que utiliza a cultura do milho proporciona aumentos na colonização micorrízica das raízes.

O sistema plantio direto com cultivo de milheto como planta de cobertura para o cultivo de milho acarreta em maior densidade de esporos de FMAs no solo. Os sistemas de preparo convencional do solo e pousio acarretam em menores valores de densidade de esporos de FMAs no solo para a cultura do milho.

A colonização micorrízica da cultura da soja e do milho na área de Cerrados foi alta, sugerindo grande utilização desta simbiose por essas culturas em sua nutrição. As comunidades de FMAs têm sua composição e diversidade influenciada pela cultura agrícola e cobertura vegetal de entressafra.

A composição da comunidade de FMAs foi diferente para os sistemas avaliados, sendo encontradas 18 espécies de FMAs, destacando-se os gêneros Acaulospora, Gigaspora, Scutellospora e Glomus. O sistema de preparo convencional do solo apresentou o menor número de espécies de FMAs em relação às coberturas de milheto e braquiária, em sistema plantio direto com milho e soja

No presente trabalho, a análise de componentes principais revelou que o uso dos FMAs e suas variáveis foram mais eficientes para evidenciar diferenças entre áreas de cultivo em sistema plantio direto sob solo de Cerrado do que as variáveis relacionadas às frações da matéria orgânica do solo.

\section{Agradecimentos}

Os autores agradecem o apoio da Fundação Agrisus, IFET-Uberaba, CNPq e CAPES.

\section{Referências}

AFEK, U.; RINALDELli, E.; MENGE, J. A.; JOHNSON, E. L. V.; POND. E. Mycorrhizal inoculum influence colonization of cotton, onion and pepper seedlings. Journal of the American Society for Horticultural Science, Alexandria, v. 115, n. 6, p. 938942, 1990.

ALGUACIL, M. M.; LUMINI, E.; ROLDÁN, A.; SALINAS-GARCÍA， J. R.; BONFANTE, P.; BIANCIOTTO, $\mathrm{V}$. The impacto $\mathrm{g}$ tillage practices on arbuscular mycorrhizal fungal diversity in subtropical crops. Ecological Applications, Washington D. C., v. 18, n. 2, p. 527-536, 2008.

ANDERSON, R. L. Diversity and no-till: keys for pestmanagement in the U.S. Great Plains. Weed Science, Champaign, v. 56, n. 1, p. 141-145, 2008.

ANTONIOLLI, Z. I.; FACELLI, E.; O' CONNOR, P.; MILLER, D.; OPHEL-KELLER, K.; SMITH, S. Spore communities of arbuscular mycorrhizal fungi and mycorrhizal associations in different ecosystems, South Australia. Revista Brasileira de Ciência do Solo, Viçosa, MG, v. 26, n. 3, p. 627-635, 2002.

AUGÉ, R. M.; STODOLA, A. J. W.; TIMS, J. E.; SAXTON, A. M. Moisture retention properties of a mycorrhizal soil. Plant and Soil, The Hague, v. 230, n. 1, p. 87-97, 2001. 
BABALOLA, O.; OSHUNSANYA, S. O.; ARE, K. Effects of vetiver grass (Vetiveria nigritana) strips, vetiver grass mulch and an organomineral fertilizer on soil, water and nutrient losses and maize yields. Soil and Tillage Research, v. 96, n. 1-2, p. 6-18, 2007.

BAYER, C.; SPAGNOLLO, E.; WILDNER, L. P.; ERNANI, P. R.; ALBUQUERQUE, J. A. Incremento de carbono e nitrogênio num Latossolo pelo uso de plantas estivais para cobertura do solo. Ciência Rural, Santa Maria, v. 33, n. 3, p. 469-475, 2003.

BÉCARD, G.; PICHÉ Y. Fungal growth stimulation by $\mathrm{CO}_{2}$ and roots exudates in vesicular-arbuscular mycorrhizal symbiosis. Applied and Environmental Microbiology, Washington D. C., v. 55, n. 9, p. 23202325, 1989.

BENEDETTI, T.; ANTONIOLLI, Z. I.; GIRACCA, E. M. N.; STEFFEN, R. B. Diversidade de fungos micorrízicos arbusculares na cultura do milho após uso de espécies de plantas de cobertura de solo. Revista de Ciencias Agroveterinárias, Lages, v. 4, n. 1, p. 44-51, 2005.

BERBARA, R. L. L.; SOUZA, F. A. de; FONSECA, H. M. A. C. Fungos Micorrízicos arbusculares: muito além da nutrição. In: FERNANDES, M. S. (Ed.). Nutrição mineral de plantas. Viçosa, MG: Sociedade Brasileira de Ciência do Solo, 2006. p. 53-88.

CAMPOS, D. T. S.; ANDRADE, J. A. C.; CASSIOLATO, A. N. R. Crescimento e micorrização de genótipos de milho em casa de vegetação. Bragantia, Campinas, v. 69, n. 3, p. 552-569, 2010.

CARNEIRO, M. A. C.; SIQUEIRA, J. O.; VALE, F. R.; CURI, N. Limitação nutricional e efeito do pré-cultivo do solo com Brachiaria decumbens e da inoculação com Glomus etunicatum no crescimento de mudas de espécies arbóreas em solo degradado. Ciência e Prática, Lavras, v. 19, n. 3, p. 281-288, 1995.

CARVALHO, D. F.; MONTBELLER, C. A.; CRUZ, E. S.; LANA, A. M. Q.; SILVA, W. A. Efeito da cobertura morta e do preparo do terreno nas perdas de solo e água em um Argissolo Vermelho-Amarelo. Engenharia na Agricultura, v. 11, n. 1-4, p. 15-22, 2003.

CORDEIRO, M. A. S.; CARNEIRO, M. A. C.; PAULINO, H. B.; SAGGIN JÚNIOR, O. J. Colonização e densidade de esporos de fungos micorrízicos em dois solos de cerrado sob diferentes sistemas de manejo. Pesquisa Agropecuária Tropical, Goiânia, v. 35, n. 3, p. 147-153, 2005.

CORKIDI, L.; ROWLAND, D. L.; JOHNSON, N. C.; ALLEN, E. B. Nitrogen fertilization alters the functioning of arbuscular mycorrhizas at two semiarid grasslands. Plant and Soil, The Hague, v. 240, n. 2, p. 299-310, 2002.
DANIELS-HETRICK, B. A.; BLOOM, J. The influence of host plant on production and colonization ability of vesicular-arbuscular mycorrhizal spores. Mycologia, New York, v. 78, n. 1, p. 32-36, 1986.

DENARDIN, J. E.; KOCHHANN, R. A.; FRAGANELLO, A.; SATTLER, A.; MANHAGO, D. D. Vertical mulching como prática conservacionista para manejo de enxurrada em sistema plantio direto. Revista Brasileira de Ciência do Solo, Viçosa, MG, v. 32, p. 2847-2852, 2008. Especial.

DIEKOW, J.; MIELNICZUK, J.; GONZÁLES-VILA, F. J.; KNICKER, H.; BAYER, C. No-till cropping systems and $\mathrm{N}$ fertilization influences on organic matter composition of physical fractions of a subtropical Acrisol as assessed by analytical pyrolysis (Py-GC/MS). Geoderma, Amsterdam, v. 135, n. 1, p. 260-268, 2006.

DIEKOW，J.; MIELNICZUK，J.; KNICKER， H.; BAYER, C.; DICK, D.P.; KÖGEL-KNABNER, I. Soil C and $\mathrm{N}$ stocks as affected by cropping systems and nitrogen fertilization in a southern Brazil Acrisol managed under no-tillage for 17 year. Soil Tillage Research, Amsterdam, v. 81, n. 1, p. 87-95, 2005.

EMPRESA BRASILEIRA DE PESQUISA AGROPECUÁRIA - EMBRAPA. Centro Nacional de Pesquisa Agropecuária de Solos (Rio de Janeiro). Manual de métodos de análise de solo. 2. ed. Rio de Janeiro: Embrapa, 1997, 212 p.

Sistema brasileiro de classificação de solos. Rio de Janeiro: Embrapa, 2006. 306 p.

FARIA, T. M.; GOMES JÚNIOR, F. G.; SÁ, M. E.; CASSIOLATO, A. M. R. Efeitos alelopáticos de extratos vegetais na germinação, colonização micorrízica e crescimento inicial de milho, soja e feijão. Revista Brasileira de Ciência do Solo, Viçosa, MG, v. 33, n. 6, p. 1624-1633, 2009.

FERNANDES, S. A. P. Avaliação de parâmetros químicos e biológicos em diferentes sistemas de manejo do solo. 1995. Dissertação (Mestrado em Microbiologia Agrícola) - Universidade de São Paulo/CENA, Piracicaba.

FIGUEIREDO, C. C.; RESCK, D. V. S.; GOMES, A. S.; FERREIRA, E. A.; RAMOS, M. L. G. Carbono e nitrogênio da biomassa microbiana em resposta a diferentes sistemas de manejo em um Latossolo Vermelho no Cerrado. Revista Brasileira de Ciência do Solo, Viçosa, MG, v. 31, n. 3, p. 551-562, 2007.

FONTANA, A.; LOSS, A.; PEREIRA, M. G.; CUNHA, T. J. F.; SALTON, J. C. Atributos de fertilidade e frações húmicas de um Latossolo Vermelho no Cerrado. Pesquisa Agropecuária Brasileira, Brasília, v. 41, n. 5, p. 847-853, 2006. 
GERDEMANN, J. W.; NICOLSON, T. H. Spores of mycorrhizal endogone species extracted from soil by wetsieving and decanting. Transactions of the British Mycological Society, Londres, v. 46, n. 2, p. 235-244, 1963.

GOMES, B. V.; CURI, N.; MOTTA, P. E. F.; KER, J. C.; MARQUES, J. J. G. S. M.; SCHULZE, D. G. Análise de componentes principais de atributos físicos, químicos e mineralógicos de solos do bioma cerrado. Revista Brasileira de Ciência do Solo, Viçosa, MG, v. 28, n. 4, p. 137-153, 2004.

GRACE, C.; STRIBLEY, D. P. A. A safer procedure for roution staining of vesicular-arbuscular mycorrhizal fungi. Mycorrizhal Research, Cambridge, v. 95, n. 10, p. 1160-1162, 1991.

JEFFRIES, P.; BAREA, J. M. Arbuscular mycorrhiza: a key component of sustainable plantsoil ecosystems. In: HOCK, B. (Ed.). The mycota. Nova Iorque: Springer, 2001. v. 9, p. 95-113.

JENKINS, W. R. A rapid centrifugal-flotation technique for separating nematodes from soil. Plant Disease, St. Paul, v. 48, n. 1, p. 692, 1964.

KABIR, Z. Tillage or no-tillage: impact on mycorrhizae. Canadian Journal of Plant Science, Ottawa, v. 85, n. 1, p. 23-29, 2005.

KITT, D. G.; DANIELS-HETRICK, B. A.; WILSON, G. T. Sporulation of two vesicular-arbuscular mycorrhizal fungi in non-sterile soil. Mycologia, Nova Iorque, v. 79, n. 6, p. 896-899, 1987.

KOSKE, R. E.; GEMMA, J. N. Amodified procedure for sting roots to detect VA mycorrhizas. Mycorrizhal Research, Cambridge, v. 92, n. 4, p. 486-488, 1989.

LA SCALA JUNIOR, N.; BOLONHEZI, D.; PEREIRA, G. T. Short-term soil CO2 emission after conventional and reduced tillage of a no till sugar cane area in Southern Brazil. Soil Tillage Research, Amsterdam, v. 91, n. 1-2, p. 244-248, 2006.

LI, Q.; CHEN, Y.; LIU, M.; ZHOU, X.; YU, S.; DONG, B. Effects of irrigation and straw mulching on microclimate characteristics and water use efficiency of winter wheat in north China. Plant Production Science, Tóquio, v. 11, n. 2, p. 161-170, 2008.

LOSS, A. Dinâmica da matéria orgânica, fertilidade e agregação do solo em áreas sob diferentes sistemas de uso no Cerrado goiano. 2011. Tese (Doutorado em Agronomia - Ciência do Solo) - Universidade Federal Rural do Rio de Janeiro, Seropédica.

LOSS, A.; ANGEliNI, G. A. R.; PEREIRA, A. C. C.; LÃ, O. R.; MAGALHÃES, M. O. L.; SILVA, E. M. R.;
SAGGIN JUNIOR, O. J. Atributos químicos do solo e ocorrência de fungos micorrízicos sob áreas de pastagem e sistema agroflorestal, Brasil. Acta Agronómica, Bogotá, v. 58, n. 2, p. 91-95, 2009a.

LOSS, A.; PEREIRA, M. G.; SCHULTZ, N.; ANJOS, L. H. C.; SILVA, E. M. R. Atributos químicos e físicos de um Argissolo Vermelho-Amarelo em sistema integrado de produção agroecológica. Pesquisa Agropecuária Brasileira, Brasília, v. 44, n. 1, p. 68-75, 2009 b.

LOSS, A.; PEREIRA, M. G.; ANJOS, L. H. C.; GIACOMO, S. G.; PERIN, A. Agregação, carbono e nitrogênio em agregados do solo sob plantio direto com integração lavoura-pecuária. Pesquisa Agropecuária Brasileira, Brasília, v. 46, n. 1, p. 648-658, 2011.

McGONIGLE, T. P.; FITTER, A. H. Ecological specificity of vesicular arbuscular mycorrhizal associations. Mycorrhizal Research, Cambridge, v. 94, n. 1, p. 120122, 1990.

McGONIGLE, T. P.; MILLER, M. H. Mycorrhizal, phosphorus absorption and yield of maize in response to tillage. Soil Science Society of America Journal, Seattle, v. 60, n. 6, p. 1856-1861, 1996.

MIRANDA, E. M.; SILVA, E. M. R.; SAGIN JUNIOR, O. J. Comunidades de fungos micorrízicos arbusculares associados ao amendoim forrageiro em pastagens consorciadas no Estado do Acre, Brasil. Acta Amazônica, Rio Branco, v. 40, n. 1, p. 13-22, 2010.

MORETI, D.; ALVES, M. C.; FILHO, W. V. V.; CARVALHO, M. P. Atributos químicos de um Latossolo Vermelho sob diferentes sistemas de preparo, adubações e plantas de cobertura. Revista Brasileira de Ciência do Solo, Viçosa, MG, v. 31, n. 1, p. 167-175, 2007.

MORTON, J. B.; BENNY, G. L. Revised classification of arbuscular mycorrhizal fungi (zygomycetes): a new order, glomales, two new suborders, glomineae and gigasporineae, with an emendations of glomaceae. Mycotaxon, EUA, v. 37, n. 1, p. 471-491, 1990.

PEREIRA, M. G.; LOSS, A.; BEUTLER, S. J.; TORRES, J. L. R. Carbono, matéria orgânica leve e fósforo remanescente em áreas de Cerrado sob plantio direto, MG. Pesquisa Agropecuária Brasileira, Brasília, v. 45 , n. 5 , p. 508-514, 2010.

PEREIRA, M. G.; LOSS, A.; BEUTLER, S. J.; TORRES, J. L. R. Granulometric and humic fractions carbon stocks of soil organic matter under no-tillage system in Uberaba, Brazil. Tropical and Subtropical Agroecossytens, Yucatán, v. 15, n. 1, p. 1-13, 2012.

RESCK, D. V. S.; FERREIRA, E. A. B.; FIGUEIREDO, C. C.; ZINN, Y. L. Dinâmica da matéria orgânica no Cerrado. In: SANTOS, G. A.; SILVA, L. S.; CANELLAS, 
L. P.; CAMARGO, F. O. (Ed.). Fundamentos da matéria orgânica do solo: ecossistemas tropicais e subtropicais. 2. ed. Porto Alegre: Metrópole, 2008. p. 359-417.

ROSA, D. M.; NOBREGA, L. H. P.; LIMA, G. P. de; MAULI, M. M. Desempenho da cultura do milho implantada sobre resíduos culturais de leguminosas de verão em sistema plantio direto. Semina: Ciências Agrárias, Londrina, v. 32, n. 4, p. 1287-1296, 2011.

SAS INSTITUTE. SAS/STAT User's guide, version 8. Cary: SAS Institute, 1999.

SCHENK, N. C.; PEREZ, Y. Manual for the identification of VA mycorrhizal fungi. Gainesville: INVAM University of Florida, 1987. 245 p.

SCHNEIDER, J.; KLAUBERG FILHO, O.; FONTOURA, S. M.; ALVES, M. V. Influência de diferentes sistemas de manejo e calagem em experimento de longa duração sobre fungos micorrízicos arbusculares. Ciência e Agrotecnologia, Lavras, v. 35, n. 4, p. 701-709, 2011.

SILVA, A. A.; GALON, R.; GALON, L.; FERREIRA, F. A.; TIRLONI, S. P.; FERRREIRA, E. A.; SILVA, A. F.AGNES, E. L. Sistema de plantio direto na palhada e seu impacto na agricultura brasileira. Revista Ceres, Viçosa, MG, v. 56, n. 306, p. 496-506, 2009.

SILVEIRA, A. P. D.; FREITAS, S. S. Microbiota do solo e qualidade ambiental. Campinas: Instituto Agronômico, 2007. $312 \mathrm{p}$.

SIQUEIRA, J. O.; FRANCO, A. A. Biotecnologia do solo: fundamentos e perspectivas. Brasília: MEC/ ABEAS/Lavras: ESAL/FAEPE, 1988. 236 p.

SIQUEIRA NETO, M.; M.; PICCOLO, M. C.; SCOPEL, E.; COSTA JUNIOR, C.; CERRI, C. C.; BERNOUX, M. Carbono total e atributos químicos com diferentes usos do solo no Cerrado. Acta Scientiarum. Agronomy, Maringá, v. 31, n. 4, p. 709-717, 2009.

SIQUEIRA, J. O.; KLAUBERG-FILHO, O. Micorrizas arbusculares: a pesquisa brasileira em perspectiva. In: NOVAIS, R. F.; ALVAREZ, V. H.; SCHAEFER, C. E. G. R. (Ed.). I Tópicos em ciências do solo. Viçosa SBCS, 2000. p. 235-259.

SMITH, S. E.; GIANINAZZI-PEARSON, V. Physiological interactions between symbionts in vesicular-arbuscular mycorrhizal plants. Annual Review of Plant Physiology and Plant Molecular Biology, Palo Alto, CA, v. 39, n. 1, p. 221-244, 1988.

SOUZA, V. C.; SILVA, R.; CARDOSO, G. D.; BARRETO, A. F. Estudos sobre fungos micorrízicos. Revista Brasileira de Engenharia Agrícola e Ambiental, Campina Grande, v. 10, n. 3, p. 612-618, 2006.

STÜRMER, S. L.; SIQUEIRA, J. O. Diversity of arbuscular mycorrhizal fungi in brazilian ecosystems. In: MOREIRA, F. M. S.; SIQUEIRA, J. O.; BRUSSAARD, L. (Ed.). Soil Biodiversity in Amazonian and other brazilian ecosystems. London: CABI Publishing, 2005. cap. 10, p. 206-236.

TORRES, J. L. R.; PEREIRA, M. G.; FABIAN, A. J. Produção de fitomassa por plantas de cobertura e mineralização de seus resíduos em plantio direto. Pesquisa Agropecuária Brasileira, Brasília, v. 43, n. 3, p. 421-428, 2008.

TORRES, J. L. R.; PEREIRA, M. G.; ANDRIOLI, I.; POLIDORO, J. C.; FABIAN, A. J. Decomposição e liberação de nitrogênio de resíduos culturais de plantas de cobertura em um solo de cerrado. Revista Brasileira de Ciência do Solo, Viçosa, MG, v. 29, n. 4, p. 609-618, 2005.

VAN DER HEIJDEN, E. W.; KUYPER, T. W. Ecological strategies of ectomycorrhizal fungi of Salix repens: root manipulation versus root replacement. Oikos, Noruega, v. 103, n. 3, p. 668-680, 2003.

VAN DER HEIJDEN, M. G. A.; KLIRONOMOS, J. N.; URSIC, M.; MOUTOGLIS, P.; STREITWOLFENGEL, R.; BOLLER, T.; WIEMKEN, A.; SANDERS, I. R. Mycorrhizal fungal diversity determines plant biodiversity, ecosystem variability and plant productivity. Nature, London, v. 396, p. 69-72, 1998.

WOHLENBERG, E. V.; REICHERT, J. M.; REINERT, D. J.; BLUME, E. Dinâmica da agregação de um solo franco-arenoso em cinco sistemas de culturas em rotação e em sucessão. Revista Brasileira de Ciência do Solo, Viçosa, MG, v. 28, n. 5, p. 891-900, 2004.

ZHANG, X.; CHEN, S.; LIU, M.; SUN, H. Improved water use efficiency associated with cultivars and agronomic management in the North China Plain. Agronomy Journal, Madison, v. 97, n. 3, p. 783-790, 2005. 